\title{
A randomized trial of a prenatal genetic testing interactive computerized information aid
}

\author{
Lynn M. YEE, MD, MPH ${ }^{1}$, Michael WOLF, PhD, MPH${ }^{2}$, Ms. Rebecca MULLEN ${ }^{2}$, Ms. Ashley R. \\ BERGERON, MPH ${ }^{2}$, Stacy Cooper BAILEY, PhD, MPH $^{3}$, Robert LEVINE, MD ${ }^{4}$, and William \\ GROBMAN, MD, MBA ${ }^{1}$ \\ ${ }^{1}$ Department of Obstetrics and Gynecology, Northwestern University Feinberg School of \\ Medicine, Chicago, IL \\ ${ }^{2}$ Department of Medicine, Northwestern University Feinberg School of Medicine, Chicago, IL \\ ${ }^{3}$ Previously with Department of Medicine, Northwestern University Feinberg School of Medicine, \\ Chicago, IL; currently, Division of Pharmaceutical Outcomes and Policy, University of North \\ Carolina Eshelman School of Pharmacy, Chapel Hill, NC \\ ${ }^{4}$ ArchieMD, Inc, Boca Raton, FL
}

\section{Abstract}

OBJECTIVE-To determine whether an interactive computer program could improve patient knowledge regarding genetic screening and diagnostic concepts.

METHODS-In this randomized trial, women 6-26 weeks' gestation were assigned to standard care with provider-based counseling or to augmented counseling with an interactive computer program. The computer-based tool conveyed information about genetic testing options. Women were administered a 23-item test of content knowledge immediately and 2-4 weeks after exposure. Test scores were compared between groups at both points using T-tests.

RESULTS-150 women were randomized equally between groups. Groups were similar with regard to demographic characteristics. Women randomized to the interactive tool correctly answered a significantly greater proportion of questions than those who received standard counseling $(69.4 \% \pm 14.2 \%$ vs. $46.0 \% \pm 15.2 \%, \mathrm{p}<.001)$ on the immediate questionnaire. One hundred and twenty-three (82\%) participants participated in the follow-up test. Women randomized to the tool continued to correctly answer a significantly greater proportion of questions $(60.6 \% \pm 16 \%$ vs. $49.7 \% \pm 18.9 \%, \mathrm{p}=.001)$. Education, health literacy, electronic health literacy, and other discussions with providers were not associated with a differential benefit from the educational intervention.

CORRESPONDING AUTHOR: Lynn M. Yee, MD, MPH, Division of Maternal-Fetal Medicine, Department of Obstetrics and Gynecology, Northwestern University Feinberg School of Medicine, 250 E. Superior Street, \#5-2175, Chicago, IL 60611, 312-472-4649, 1ynn.yee@ northwestern.edu.

This abstract was presented as an oral presentation (abstract \#21) at the 2012 Society of Maternal-Fetal Medicine $32^{\text {nd }}$ Annual Meeting in Dallas, Texas.

DISCLOSURES: Robert Levine is the President of ArchieMD. All other authors report no conflicts of interest. 
CONCLUSION-A patient-directed interactive computer program may help providers to convey relevant information about genetic screening and diagnostic concepts.

\section{INTRODUCTION}

Prenatal screening and diagnosis can provide pregnant women with important information. However, testing involves varying degrees of uncertainty and risk, options are increasingly complex, and patient knowledge of available tests and their risks is generally low. ${ }^{1-3}$ Fully informed decisions regarding prenatal screening and diagnosis require a significant degree of patient education. Direct provider counseling may be insufficient for patients to fully comprehend such complex information, particularly within the context of a brief clinical encounter. ${ }^{1-8}$ Counseling may also be limited due to factors such as provider bias or limitations in provider knowledge. Further, women with low levels of education and/or health literacy are particularly likely to have inadequate understanding of prenatal genetic testing. ${ }^{9}$

Information and decision aids have been recommended for use in clinical situations in which patient education is necessary for fully informed decision-making. ${ }^{10-13}$ Use of decision aids for prenatal counseling using structured, paper-based or basic electronic tools has been associated with less decision conflict, improved knowledge, and in some cases, increased uptake of prenatal testing. ${ }^{14-18}$ Such tools can facilitate an improved informed consent process, yet few decision aids have been developed and evaluated in the United States, particularly for use in low literacy populations. ${ }^{11}$ In 2009, Kuppermann et al showed that use of an interactive prenatal diagnostic decision tool resulted in better patient satisfaction, more decision certainty, and more accurate estimates of risk among women receiving prenatal genetic counseling. ${ }^{19}$ However, this tool was not developed for a low literacy population and compared the computerized tool to a standardized written educational booklet.

In this study, we aimed to develop and test an information aid that used the latest interactive computer technology to provide an immersive educational experience about prenatal genetic testing options. This tool, developed by ArchieMD (Boca Raton, FL), incorporates 3D medical models and visualizations to convey complex information about medical options and procedures. The primary goal of the study was to determine whether women who received the ArchieMD Interactive Informed Consent and Education Program for Pregnant Women had improved understanding and knowledge of prenatal screening compared to women who received standard prenatal care. We hypothesized that use of the interactive education tool in a prenatal genetic testing setting would improve patient understanding.

\section{METHODS}

This was a randomized controlled trial comparing a treatment group receiving an interactive patient education tool for prenatal screening and diagnosis to a control group receiving standard of care counseling. This interactive tool was developed with several principles in mind. State-of-the-art technology enabled the user to view the body internally via 3D computer models of the body. The program included a "virtual guide" for clarification of 
complex medical terms and the capacity to type notes and questions for later discussion. User involvement was encouraged by providing an avatar who could be directed by the participant to further explore topics of interest or re-explore topics when desired. The modules included information on basic prenatal testing concepts, anatomy, common genetic abnormalities, specific information about invasive and non-invasive test options, including chorionic villous sampling (CVS) and amniocentesis, and the risks, benefits and alternatives to these tests. This tool was developed before the clinical availability of cell-free fetal DNA testing and thus did not include information about this modality. Experts in health literacy tailored language to ensure it was appropriate for women of low and high health literacy; best practices were used to convey numerical concepts. ${ }^{20}$ Participants had the opportunity to demonstrate comprehension of seminal concepts by replying to questions and receiving immediate feedback. Participants could spend varying amounts of time using the tool depending on their degree of user involvement.

After approval from the Northwestern University Institutional Review Board, pregnant women (>18 years) were recruited during routine prenatal visits; women were not recruited at genetic counseling sessions. The study procedures took place at the site of prenatal care. Eligibility criteria included gestational age between 6 and 26 weeks, having not yet undergone any prenatal testing, and ability to speak English. Women carrying multiple gestations were excluded. Women who agreed to participate provided written, informed consent. This trial was registered at Clinicaltrials.gov (NCT01176019) prior to its initiation.

Participants were administered an in-person, computer-based questionnaire that included questions about demographic characteristics and two validated scales that assessed health literacy and electronic health literacy. Health literacy was assessed via the Rapid Estimate of Adult Literacy in Medicine (REALM), a widely used health literacy word recognition and pronunciation test that is closely validated with reading comprehension tests. ${ }^{21}$ The eHealth Literacy Scale (eHEALS) is an 8-item scale (score range 8 to 40) that was administered to assess computer literacy as related to health information. ${ }^{22}$ Electronic health literacy reflects perceived skills at finding, evaluating, and applying electronic health information; higher scores indicate better perceived electronic health literacy.

Participants were randomized using a computer-generated random number table. Women in the standard care arm did not receive any additional counseling beyond the antenatal counseling they had already received during their clinic visit. Women in the intervention arm received the standard care counseling and the interactive education tool. Women undergoing the intervention completed the self-guided computerized information aid in a private clinic room with a research assistant available for questions and assistance. Women could spend as much time with the tool as they desired. Women in the standard of care arm were directed immediately to the post-test. Thus, immediately after the randomized exposure, both groups were administered a 23-item test designed to assess knowledge of the concepts of prenatal screening and diagnosis. This test asked 18 true-false and 3 open-ended questions. Test contents are provided in the supplementary material. The open-ended questions asked women to name two tests that could definitively give chromosomal information, name two types of abnormalities that could be identified with CVS or amniocentesis, and name one potential complication of these tests. The true/false questions 
were content-based questions designed to directly test basic knowledge about genetic screening and testing. Women randomized to the educational tool group were additionally asked their opinions of the tool using visual analog scales. Two to four weeks after randomization the subjects were re-contacted and administered the same questionnaire, via a telephone interview, to measure longer-term knowledge retention.

The 23-item knowledge test was scored as a proportion of correct answers. Open-ended questions were judged to be correct by a single investigator (WAG) who was unaware of a participants' group assignment. The sample size of 150 was determined based on an alpha of 0.05 , and $80 \%$ power to detect at least a $7 \%$ improvement in correct responses on this questionnaire (with a standard deviation of $15 \%$ ) after use of the interactive education tool. ${ }^{23}$ Student t-tests were used for group comparisons and two-way ANOVA was used to assess whether there was an interaction between group assignment and patient educational level, health literacy, electronic health literacy, and prior discussion about genetic topics with a provider. All tests were two-tailed and $\mathrm{p}<.05$ was used to define statistical significance. Analyses were performed with Minitab 14 (Minitab, Inc., State College, PA).

\section{RESULTS}

From August 2010 to March 2011, two hundred and sixty-one eligible women were approached for participation. Of these, 111 declined. Of the 150 women who participated, 75 were randomized to each group (Figure 1). Table 1 demonstrates their demographic characteristics. Groups did not differ by age, gestational age, parity, race/ethnicity, or education. The proportion of women with limited health literacy was over 40\%. eHEALS scores were not different by group and indicated a high degree of comfort with electronic health information among women who consented to participate.

Women randomized to the interactive education tool correctly answered a mean of $69.4 \%$ $( \pm 14.2 \%)$ of questions correctly on the initial test evaluating knowledge of prenatal diagnostic concepts. This score was significantly greater than the mean of $46.0 \%( \pm 15.2 \%)$ correct answers among the group of women who only received standard counseling $(\mathrm{p}<0.001)$. Eighty-two percent $(\mathrm{N}=123)$ of women were successfully contacted at a median of 23 days after randomization and completed the questionnaire again (64 [85\%] in the control group vs $59[79 \%]$ in the intervention group, $\mathrm{p}=0.29)$. As before, the group who had received the interactive education tool had a significantly higher mean score than the group who had not been exposed to the tool $(60.6 \% \pm 16.0 \%$ vs. $49.7 \% \pm 18.9 \%, \mathrm{p}=0.001)$.

Subgroup analyses were then performed to assess whether the benefits associated with exposure to the interactive education tool were affected by education level, health literacy, electronic health literacy, and recall of prenatal diagnostic counseling by a health care provider (Table 2). Regardless of educational attainment, health literacy, or electronic health literacy, women exposed to the interactive education tool demonstrated a similar improvement in scores compared to women in the standard care group. In addition, over $80 \%$ of participants in each group recalled having received prenatal diagnostic counseling with their provider during the current pregnancy but prior to randomization. However, recall of having received such counseling did not alter the improvement in scores on the initial 
questionnaire among women in the educational intervention arm compared to the control group (test for interaction $\mathrm{p}=0.19$ ). After randomization, but prior to follow-up, $48.8 \%$ ( $\mathrm{N}=60$ of 123 available for follow-up) of women reported receiving additional counseling as a part of their routine prenatal care. Subsequent provider counseling after randomization did not diminish the relative benefit that accrued to those who had been exposed to the education tool. Finally, women receiving the interactive education tool were asked their opinions of the tool. All women (100\%) who experienced the education tool thought it was helpful. A majority also felt the tool was balanced (77\%), clear (96\%) and had the right quantity of information (77\%). Of the women who felt the tool did not have the right quantity of information, the majority (88\%) felt it had too much information.

\section{COMMENT}

We found that women exposed to standard counseling plus a prenatal diagnostic educational tool that incorporated interactive, state-of-the-art technology demonstrated improved knowledge of prenatal genetic testing options and better retention of such information compared to women who received only standard counseling. A similar magnitude of improvement was obtained regardless of education level and health literacy. Similarly, the benefit was not dependent on having great facility with electronic resources, as there was no interaction with electronic health literacy. Further this effect did not appear dependent on women having not received prenatal diagnostic counseling from their provider. Over $80 \%$ of women had specific recollection of a session involving prenatal counseling during their antenatal care prior to randomization, and the presence of such a session prior to or after being exposed to the counseling tool did not affect the demonstrated benefit. Finally, women exposed to the computer-based, interactive tool found it to be clear, balanced and helpful.

Prior studies report a decrease in decision conflict following use of an information or decision aid. ${ }^{11}$ In our study, high participant satisfaction coupled with the observed improvement in knowledge about these complex topics suggest such a tool is both acceptable to participants and promotes knowledge acquisition better than when standard provider-based counseling is the sole form of information transfer. One strength of this study is that it compared augmented education to actual provider-based counseling about prenatal testing options, rather than to counseling by specialized but not universally available genetic counselors. This tool educated patients on topics that should be addressed in prenatal care for all women but may not be consistently discussed; it enhances, rather than replaces, direct provider counseling, and may help patients and providers determine when more in-depth counseling by a specialist is needed. We would anticipate that the improvements in knowledge seen here would be reflected in more informed decision making about prenatal testing options. Although the goal of counseling is to empower more informed decisions rather than promote testing use, prior work suggests women with better knowledge were more likely to desire prenatal screening. ${ }^{18}$ We hypothesize that use of an interactive tool with balanced education of risks and benefits and attention to best practices for explanation of complex concepts may result in improved understanding of testing options; this knowledge increase may ultimately be associated with alterations in uptake of testing. Health literacy, or the skills needed to function in the health care environment, is recognized as a mediator of health disparities in the United States. ${ }^{24-26}$ Inadequate health 
literacy has a clear association with poor health outcomes, inadequate utilization of health care services, and poor health knowledge. ${ }^{24,25,27-31}$ An important strength of our study was the focus on developing an intervention appropriate for women with limited health literacy, since this population is large and particularly likely to benefit from added health education. Further, educational interventions that benefit patients in low literacy populations typically benefit individuals in higher literacy groups as well. ${ }^{32}$ Inadequate health literacy is a common public health problem, even in populations with high amounts of formal education. As shown here, education alone is inadequate to prepare women for the complex medical counseling that occurs with prenatal genetic testing, and this interactive tool was helpful for women regardless of education or literacy level.

Several limitations of this study warrant discussion. First, although the sample had racial/ ethnic diversity, it was largely a young population seeking care at a single academic center. Thus, the findings may not be generalizable to other populations. It should also be noted that the rate of study participation was $57 \%$; it is possible that the women choosing to participate were not representative of the total population approached. It was not possible to compare the enrolled population to those who declined to participate as information could not be collected on the latter group once they declined participation. Second, the field of prenatal genetic testing is rapidly evolving. Several new technologies have been introduced since the development of the interactive education tool. The tool was not intended to counsel about options such as cell-free fetal DNA non-invasive prenatal testing or microarray analysis from invasive testing specimens. However, there is no reason to believe that the further complexity of choices would make an interactive education tool any less useful. In addition, it was not possible to control for the quality or amount of genetic counseling women might have received from their providers prior to randomization, although the effect of the tool did not appear to differ based on gestational age at enrollment or memory of a specific counseling session with their provider. In addition, the heterogeneity of the population reflects the reality of prenatal care, in which women receive counseling about such topics across multiple time points and with different levels of quality. Finally, this study was not designed to evaluate actual differences in decisions made by pregnant women, as the goal of the tool was to improve knowledge rather than to change patient decisions per se. Thus, although the increase in knowledge subsequent to the interactive education tool is a promising proxy for better informed decision making compared to standard of care counseling, we did not actually measure the effects of more informed choice on patient decisions.

Prenatal genetic testing requires women and their families to make complex, value-laden decisions about their pregnancy and family goals. Providers have an obligation to provide education and non-directive counseling about these decisions, yet many current counseling efforts may not allow optimally informed decisions. We identified that women exposed to augmented prenatal diagnostic education using interactive technology demonstrate better knowledge about prenatal genetic testing compared to women who experience standard provider counseling. Women of diverse educational, health literacy, and electronic literacy backgrounds experienced this benefit, suggesting a tool such as this may be widely applicable. We encourage ongoing research at the intersection of health services, health 
literacy, and prenatal testing, in order to elucidate how women are able to optimize their decision making with regard to prenatal diagnosis.

\section{Supplementary Material}

Refer to Web version on PubMed Central for supplementary material.

\section{Acknowledgments}

FUNDING: NICHD 1R41HD058439-01A2. All work is the responsibility of the authors; the funding organization had no role in study design, analysis, or manuscript preparation.

\section{References}

1. Skirton H, Murakami K, Ito M, et al. A report of two linked studies of knowledge and attitudes to prenatal screening and testing in adults of reproductive age in Japan and the UK. Midwifery. 2008; 24:270-80. [PubMed: 17270325]

2. Jaques A, Halliday J, Bell R. Do women know that prenatal testing detects fetuses with Down syndrome? J Obstet Gynaecol. 2004; 24:647-51. [PubMed: 16147604]

3. Rowe H, Fisher J, Quinlivan J. Are pregnant Australian women well informed about prenatal genetic screening? A systematic investigation using the Multidimensional Measure of Informed Choice. Aust N Z J Obstet Gynaecol. 2006; 46:433-9. [PubMed: 16953859]

4. Dormandy E, Michie S, Hooper R, Marteau T. Informed choice in antenatal Down syndrome screening: a cluster-randomised trial of combined versus separate visit testing. Patient Educ Couns. 2006; 61:56-64. [PubMed: 16533677]

5. Gourounti K, Sandall J. Do pregnant women in Greece make informed choices about antenatal screening for Down's syndrome? A questionnaire survey. Midwifery. 2008; 24:153-62. [PubMed: 17316936]

6. Kohut R, Dewey D, Love E. Women's knowledge of prenatal ultrasound and informed choice. J Genet Couns. 2002; 11:265-76. [PubMed: 12795303]

7. Timmermans D, ten Kate L, van Vugt J, van der Wal G. Are pregnant women making informed choices about prenatal screening? Genet Med. 2005; 7:332-8. [PubMed: 15915085]

8. van den Berg M, Timmermans D, ten Kate L, et al. Informed decision making in the context of prenatal screening. Patient Educ Couns. 2006; 63:110-7. [PubMed: 16242899]

9. Cho R, Plunkett B, Wolf M, et al. Health literacy and patient understanding of screening tests for aneuploidy and neural tube defects. Prenatal Diagnosis. 2007; 27:463-7. [PubMed: 17345585]

10. Woolf S, Chan E, Harris R, et al. Promoting informed choice: transforming health care to dispense knowledge for decision-making. Ann Intern Med. 2005:143. [PubMed: 16027457]

11. Stacey D, Bennett C, Barry M, et al. Decision aids for people facing health treatment or screening decisions. The Cochrane Database of Systematic Reviews. 2011; 10:CD001431. [PubMed: 21975733]

12. Dugas M, Shorten A, Dubé E, et al. Decision aid tools to support women's decision making in pregnancy and birth: a systematic review and meta-analysis. Social Science and Medicine. 2012; 74:1968-78. [PubMed: 22475401]

13. Vlemmix F, Warendorf J, Rosman A, et al. Decision aids to improve informed decision-making in pregnancy care: a systematic review. British Journal of Obstetrics and Gynecology. 2013; 120:257-66.

14. Graham W, Smith P, Kamal A, et al. Randomised controlled trial comparing effectiveness of touch screen system with leaflet for providing women with information on prenatal tests. British Medical Journal. 2000; 320:155-60. [PubMed: 10634736]

15. Bekker H, Hewison J, Thornton J. Applying decision analysis to facilitate informed decision making about prenatal diagnosis for Down syndrome: a randomised controlled trial. Prenatal Diagnosis. 2004; 24:265-75. [PubMed: 15065100] 
16. Nagle C, Gunn J, Bell R, et al. Use of a decision aid for prenatal testing of fetal abnormalities to improve women's informed decision making: a cluster randomised controlled trial [ISRCTN22532458]. British Journal of Obstetrics and Gynecology. 2008; 115:339-47.

17. Hunter A, Cappelli M, Humphreys L, et al. A randomized trial comparing alternative approaches to prenatal diagnosis counseling in advanced maternal age patients. Clinical Genetics. 2005; 67:30313. [PubMed: 15733266]

18. Stefansdottir V, Skirton H, Jonasson K, et al. Effects of knowledge, education, and experience on acceptance of first trimester screening for chromosomal anomalies. Acta Obstet Gynecol Scand. 2010; 89:931-8. [PubMed: 20235896]

19. Kuppermann M, Norton M, Gates E, et al. Computerized prenatal genetic testing decision-assisting tool: A randomized controlled trial. Obstetrics and Gynecology. 2009; 113:53-63. [PubMed: 19104360]

20. Apter A, Paasche-Orlow M, Remillard J, et al. Numeracy and communication with patients: they are counting on us. Journal of General Internal Medicine. 2008; 23:2117-24. [PubMed: 18830764]

21. Davis T, Long S, Jackson R, et al. Rapid estimate of adult literacy in medicine: a shortened screening instrument. Family Medicine. 1993; 25:391-5. [PubMed: 8349060]

22. Norman C, Skinner H. eHEALS: The eHealth Literacy Scale. Journal of Medical Internet Research. 2006; 8:e27. [PubMed: 17213046]

23. Tait A, Voepel-Lewis T, Malviya S, Philipson S. Improving the readability and processability of a pediatric informed consent document: effects on parents' understanding. Arch Pediatr Adolesc Med. 2005; 159:347-52. [PubMed: 15809387]

24. DeWalt DA, Berkman ND, Sheridan S, et al. Literacy and health outcomes: A systematic review of the literature. Journal of General Internal Medicine. 2004; 19:1228-39. [PubMed: 15610334]

25. Osborn CY, Cavanaugh K, Wallston KA, et al. Health literacy explains racial disparities in diabetes medication adherence. Journal of Health Communication: International Perspectives. 2011; 16:268-78.

26. Nielsen-Bohlman, L.; Panzer, AM.; Kindig, DA. Health Literacy: a Prescription to End Confusion. Washington, DC: The National Academies Press; 2004.

27. Schillinger D, Grumbach K, Piette J, et al. Association of health literacy with diabetes outcomes. Journal of the American Medical Association. 2002; 288:475-82. [PubMed: 12132978]

28. Wolf M, Knight S, Lyons E, et al. Literacy, race, and PSA level among low-income men newly diagnosed with prostate cancer. Urology. 2006; 68:89-93. [PubMed: 16844451]

29. Lindau ST, Basu A, Leitsch SA. Health literacy as a predictor of follow-up after an abnormal Pap smear: A prospective study. Journal of General Internal Medicine. 2006; 21:829-34. [PubMed: 16881942]

30. Berkman ND, Sheridan S, Donahue KE, et al. Low health literacy and health outcomes: An updated systematic review. Ann Intern Med. 2011; 155:97-107. [PubMed: 21768583]

31. Dolan N, Ferreira M, Davis T, et al. Colorectal cancer screening knowledge, attitudes, and beliefs among veterans: Does literacy make a difference? J Clin Oncol. 2004; 22:2617-22. [PubMed: 15226329]

32. You W, Wolf M, Bailey S, Grobman W. Improving patient understanding of preeclampsia: a randomized controlled trial. American Journal of Obstetrics and Gynecology. 2012; 206:e1-5. [PubMed: 22542120] 


\section{What's already known about this subject?}

- Informed decisions about prenatal genetic screening and diagnosis require complex patient education. Many women feel prenatal genetic information is difficult to understand.

- Information aids may enhance patient knowledge but have been inadequately studied in diverse populations in the United States.

\section{What does this study add?}

- Pregnant women exposed to an interactive computerized tool about prenatal genetic screening had better post-test knowledge than women receiving standard of care counseling.

- This interactive information aid enhanced knowledge regardless of health literacy, electronic health literacy, or educational level. 


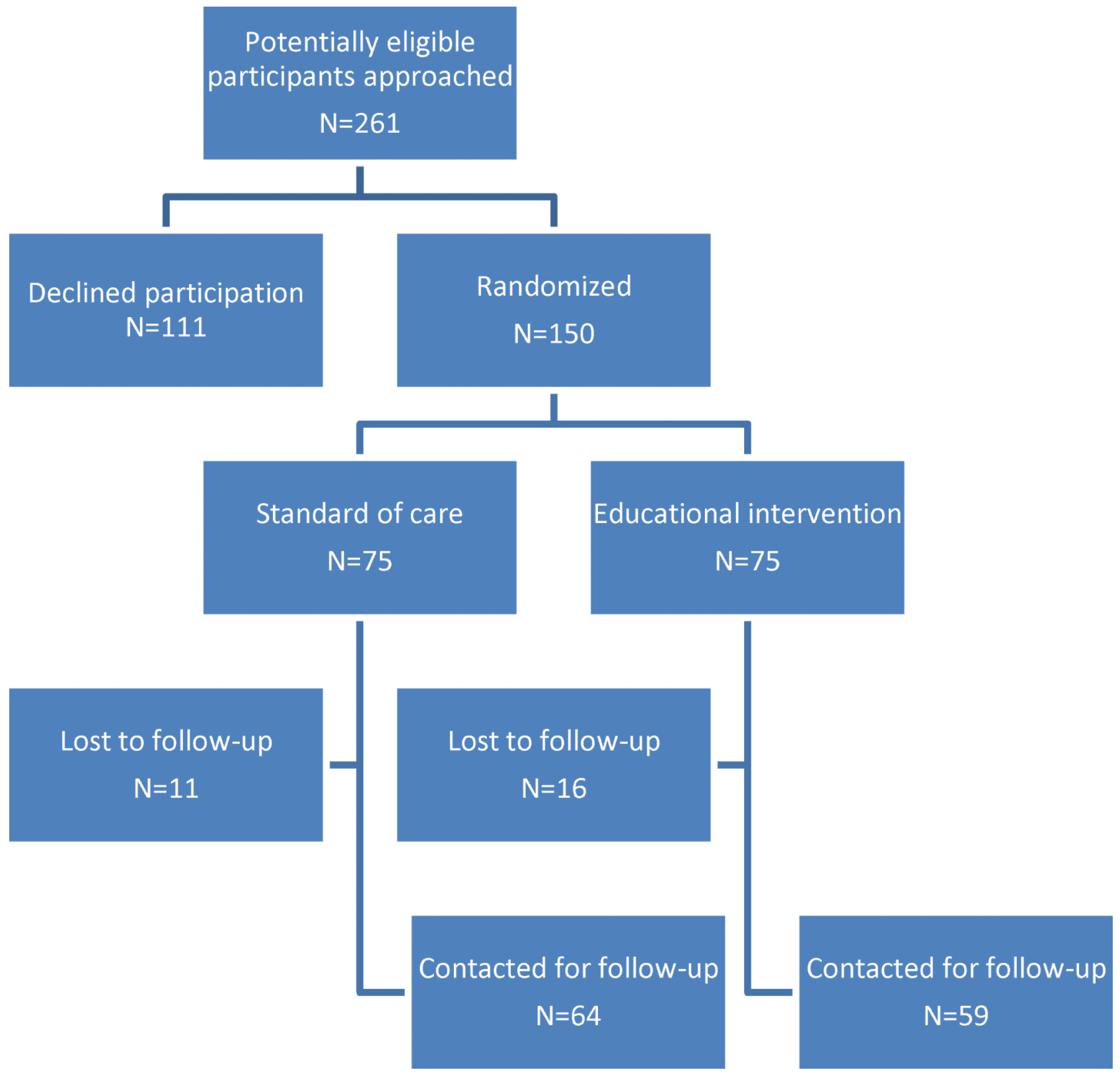

Figure 1.

Flow diagram of study participants 


\section{Table 1}

Demographic characteristics of participants

\begin{tabular}{lcccc}
\hline & All participants $(\mathbf{N}=\mathbf{1 5 0})$ & Educational tool (N=75) & Standard care $(\mathbf{N}=\mathbf{7 5})$ & p value \\
\hline Age (years) & $26.6(5.3)$ & $26.0(5.0)$ & $27.3(5.5)$ & 0.13 \\
Gestational age > 20 weeks & 45.3 & 46.7 & 44.0 & 0.74 \\
Primigravida & 15.3 & 16.0 & 14.7 & 0.82 \\
Race/Ethnicity & & & \\
Non-Hispanic White & 7.3 & 5.4 & 9.3 & 0.09 \\
Non-Hispanic Black & 53.3 & 45.3 & 61.4 & \\
Hispanic & 28.0 & 36.0 & 20.0 & \\
Other & 11.4 & 13.3 & 9.3 & \\
Education & & & & \\
< High School & 9.8 & 9.4 & 10.2 & 30.5 \\
High School Grad/GED & 26.0 & 21.9 & 30.5 & \\
Some College & 35.8 & 40.6 & 28.8 & \\
$\quad$ College Graduate & 28.4 & 28.1 & 85.3 & 0.66 \\
Prior provider discussion & 84.0 & 82.7 & 38.7 & 0.25 \\
Limited health literacy & 43.3 & 48.0 & $30(4.8)$ & 0.78 \\
eHEALS score & $30(5)$ & $30(4.9)$ & &
\end{tabular}

All data provided as mean (standard deviation) or $\%$. 
Table 2

Initial test scores after randomized exposure by subgroup

\begin{tabular}{|c|c|c|c|}
\hline & $\begin{array}{c}\text { Standard care, \% correct, } S D \\
(\mathbf{N}=75)\end{array}$ & $\begin{array}{l}\text { Educational tool, \% correct, SD } \\
(\mathbf{N}=75)\end{array}$ & $P$ value for interaction \\
\hline Education & & & 0.1 \\
\hline Less than college & $44.9 \pm 14.9$ & $66.4 \pm 13.4$ & \\
\hline College or greater & $48.9 \pm 16.2$ & $79.2 \pm 12.3$ & \\
\hline Health literacy & & & 0.81 \\
\hline Limited & $39.7 \pm 13.7$ & $64.7 \pm 13.7$ & \\
\hline Adequate & $49.9 \pm 15.0$ & $73.8 \pm 13.3$ & \\
\hline Electronic health literacy & & & 0.74 \\
\hline Less than average & $43.8 \pm 15.0$ & $66.3 \pm 15.4$ & \\
\hline At least average & $47.4 \pm 15.4$ & $71.6 \pm 13.0$ & \\
\hline Prior provider discussion & & & 0.19 \\
\hline No & $45.9 \pm 13.7$ & $62.2 \pm 15.6$ & \\
\hline Yes & $45.9 \pm 15.6$ & $71.0 \pm 13.5$ & \\
\hline Discussion after randomization ${ }^{*}$ & & & 0.20 \\
\hline No & $48.4 \pm 19.9$ & $63.5 \pm 16.6$ & \\
\hline Yes & $51.5 \pm 17.6$ & $58.3 \pm 15.3$ & \\
\hline
\end{tabular}

